\title{
Do Biliary Complications after Hypofractionated Radiation Therapy in Hepatocellular Carcinoma Matter?
}

\section{Jeong II Yu, MD' \\ Hee Chul Park, MD, PhD \\ Do Hoon Lim, MD, PhD' \\ Seung Woon Paik, MD, PhD²}

Departments of ${ }^{1}$ Radiation Oncology and ${ }^{2}$ Medicine, Samsung Medical Center, Sungkyunkwan University

School of Medicine, Seoul, Korea
Correspondence: Hee Chul Park, MD, PhD Department of Radiation Oncology,

Samsung Medical Center,

Sungkyunkwan University School of Medicine, 81 Irwon-ro, Gangnam-gu, Seoul 06351, Korea

Tel.: 82-2-3410-2612

Fax: 82-2-3410-2619

E-mail: hee.ro.park@skku.edu

Received February 23, 2015

Accepted June 29, 2015

Published Online July 17, 2015

\section{Purpose}

The purpose of this study is to evaluate the efficacy of hypofractionated radiation therapy (RT) in the treatment of unresectable hepatocellular carcinoma (HCC) after failure of transarterial chemoembolization (TACE) or in cases of refractory HCC, and to investigate biliary complications after hypofractionated RT.

\section{Materials and Methods}

We retrospectively enrolled patients with unresectable, TACE-unresponsive, or refractory HCC treated with hypofractionated RT between July 2006 and December 2012. The perihilar region was defined as the $1-\mathrm{cm}$ area surrounding the right, left, and the common hepatic duct, including the gallbladder and the cystic duct. Significant elevation of total bilirubin was defined as an increase of more than $3.0 \mathrm{mg} / \mathrm{dL}$, and more than two times that of the previous level after completion of RT.

\section{Results}

Fifty patients received hypofractionated RT and 27 (54\%) had a tumor located within the perihilar region. The median follow-up period was 24.7 months (range, 4.3 to 95.5 months). None of the patients developed classic radiation disease symptoms, but four patients (8\%) showed significant elevation of total bilirubin within 1 year after RT. During follow-up, 12 patients (24\%) developed radiologic biliary abnormalities, but only two patients had toxicities requiring intervention. Estimated local progression-free survival, progression-free survival, and overall survival of the patients at 3-year post-hypofractionated RT were $89.7 \%$, $11.2 \%$, and $57.4 \%$, respectively.

\section{Conclusion}

Biliary complications associated with a higher dose exposure of hypofractionated RT were minimal, even in the perihilar region. Hypofractionated RT provided excellent local control and may be a valuable option for treatment of unresectable cases of TACE-unresponsive or refractory HCC.

\section{Introduction}

In treatment of unresectable cases of hepatocellular carcinoma (HCC), sorafenib is the only valid treatment option if trans-arterial chemoembolization (TACE) fails or has an insufficient effect [1]. In a subgroup analysis of two random-
Key words

Hepatocellular carcinoma, Radiotherapy, Dose fractionation, Toxicity 
ized controlled trials, strong evidence from retrospective and prospective studies has shown that RT can provide good local control and extend patient survival $[4,5]$. In particular, stereotactic ablative body RT (SABR) and hypofractionated RT yield an excellent objective response rate with a $>80 \%$ local control rate [6-8].

The most important concern associated with RT for HCC is deterioration of normal liver function; however, with advances in RT techniques and improved radiobiology, the classic radiation-induced liver disease (RILD) observed in the early days of RT is becoming less of a problem [9]. Nevertheless, biliary inflammation with or without stricture remains a concern in application of RT in unresectable cases of HCC. Several studies have reported on the emergence of symptomatic biliary complications 1 to 10 years after RT for tumors related to the biliary system; however, those studies were conducted more than 20 years ago [10,11].

Previously, we reported results for treatment of small, but unresectable, TACE-unresponsive or refractory HCC with hypofractionated RT [12]. In this study, we found that outcomes of hypofractionated RT were comparable to those using other RT techniques, specifically $85.0 \%$ local control and $100.0 \%$ overall survival (OS) at 2 years. However, this study was limited by its small sample size and short followup period.

To address our previous findings and limited conclusions, in this study we evaluated the efficacy of hypofractionated RT in unresectable cases of TACE-unresponsive or refractory HCC and investigated the incidence of biliary stricture after hypofractionated RT.

\section{Materials and Methods}

Patients with unresectable, TACE-unresponsive, or refractory HCC treated with hypofractionated RT at Samsung Medical Center between July 2006 and December 2012 were enrolled retrospectively. The Samsung Medical Center Institutional Review Board approved this study and exempted the requirement for written consent.

All patients enrolled in this study received hypofractionated RT for treatment of unresectable, TACE-unresponsive, or refractory HCC. The inclusion criteria were as follows: Eastern Cooperative Oncology Group (ECOG) performance score of 0 or 1 ; normal liver volume (whole liver minus gross tumor volume) of $\geq 700 \mathrm{~mL}$; HCC size $<5 \mathrm{~cm} ; \leq 15 \%$ of normal liver volume exposed to $50 \%$ of the prescribed RT dose; and tumor site not adjacent to critical organs such as the gastroduodenum or the large/small bowel.

To avoid inaccurate targeting secondary to tumor move- ment during radiation treatment, an additional margin following the cranio-caudal orientation was outlined using fluoroscopy; this was done before setting up the respirationgated protocol with four-dimensional computed tomography (CT) simulation in 2008. Since then, respiratory gating has become the routine protocol for administration of RT for HCC. The technical details of our simulation, planning, verification, and RT-delivery protocols are described in the previous report [13]. The total number of fractions was ten and the fraction size of hypofractionated RT was 5 or $6 \mathrm{~Gy}$. The plan objective was to cover at least $70 \%$ of the clinical target volume with $100 \%$ of the prescribed dose.

The first follow-up evaluations after hypofractionated RT were performed 1 month after treatment and every 2 to 3 months thereafter. At each follow-up, patients underwent clinical and physical examinations, CT or magnetic resonance imaging (MRI) with or without positron emission tomography, measurement of serum tumor markers, and chemistry profiling that included liver function tests. Patient toxicity levels were assessed using the Common Terminology Criteria for Adverse Events, ver. 4.0. The response to hypofractionated RT was evaluated using the modified response evaluation criteria for solid tumors based on CT or MRI scans acquired at the 3- to 5-month follow-up visit.

The perihilar region was defined as the area within $1 \mathrm{~cm}$ right and left of the hepatic duct and the common hepatic duct, including the gallbladder and the cystic duct. Significant elevation of total bilirubin was defined as total bilirubin elevation $>3.0 \mathrm{mg} / \mathrm{dL}$ and more than two times the previous level after RT completion. Biliary dilation was defined as duct dilatation two or more times greater than the previous diameter and / or appearance of a biliary tree that was previously unseen in the radiologic examination. If total bilirubin elevation was unreasonable based on laboratory liver function results and if biliary dilatation was noted, biliary drainage was performed via endoscopic retrograde biliary drainage (ERBD) and / or percutaneous trans-hepatic biliary drainage (PTBD).

Local progression (LP) was defined as the presence of a $\geq 20 \%$ increase in the viable tumor diameter, which was encompassed by a planning target volume in images taken during follow-up. Intra-hepatic progression (IHP) was defined as new recurrence of a viable tumor or progression of a previously controlled tumor in the liver, including vascular invasion beyond the RT field. Extra-hepatic progression (EHP) was defined as any recurrence outside the liver.

LP-free survival (LPFS), progression-free survival (PFS), and OS were calculated as the duration from the start date of receiving hypofractionated RT to the date when a new event was first detected or the date of the last follow-up visit, if follow-up had been event-free.

Fisher exact tests were used for comparison of differences 
in clinical characteristics and treatment types between the groups. The Kaplan-Meier method was used for calculation of survival curves, and the log-rank test was used for comparison of differences between the survival curves. Multivariate survival analysis was performed using a Cox proportional hazards model tested using the Schoenfeld residuals method with variables showing statistical significance in univariate analysis and Child-Pugh class. The PASW ver. 21.0 (IBM Co., Armonk, NY) was used for all analyses. All statistical tests were evaluated as two-sided and $p<0.05$ was considered statistically significant.

\section{Results}

\section{Patients}

During the study period, 50 patients received hypofractionated RT for treatment of unresectable, TACE-unresponsive, or refractory HCC. The median follow-up period for the patient samples was 24.7 months (range, 4.3 to 95.5 months).

Patient characteristics are shown in Table 1. Median patient age was 61 years (range, 38 to 83 years) and male patients accounted for more than $85 \%$ of total cases. Serum hepatitis B viral antigen markers were positive in 37 patients (74\%); 41 patients (82\%) had concurrent liver cirrhosis; and three patients $(6 \%)$ had Child-Pugh scores of class B. Twentyseven patients $(54 \%)$ had the tumor located within the perihilar region. The median $\alpha$-fetoprotein (AFP) level before RT was $46.2 \mathrm{ng} / \mathrm{mL}$ (range, 1.6 to $5,077.4 \mathrm{ng} / \mathrm{mL}$ ). Ten patients $(20 \%)$ also had portal-vein tumor thrombosis, and three patients had tumors involving bile-duct invasion. A fraction size of 6 Gy was used in five patients (10\%).

All enrolled patients had undergone TACE treatment at least once (median number of TACE treatments, 4; range, 1 to 20). TACE treatment was combined with surgical resection, radiofrequency ablation (RFA), or previous RT in 14 $(28 \%), 23(46 \%)$, and three $(6 \%)$ patients, respectively. Although exact staging was not possible because of multiple previous treatments, Barcelona Clinic for Liver Cancer (BCLC) C-stage was identified in 18 patients (36\%).

\section{Treatment response}

In the images taken at the 3- to 5-month follow-up evaluation, 27 patients (54\%) showed a complete response of the viable contrast-enhanced lesion, 11 patients $(22 \%)$ showed a partial response, and 10 patients $(20 \%)$ showed stable disease. LP within 5 months after RT was detected in two patients (4\%). Among the five patients who received a frac-
Table 1. Baseline characteristics of hepatocellular carcinoma patients

\begin{tabular}{|c|c|}
\hline Variable & $\begin{array}{l}\text { No. of patients }(\%) \\
(n=50)\end{array}$ \\
\hline Age, median (range, yr) & $61(38-83)$ \\
\hline \multicolumn{2}{|l|}{ Sex } \\
\hline Male & $43(86)$ \\
\hline Female & $7(14)$ \\
\hline \multicolumn{2}{|l|}{ ECOG performance status } \\
\hline 0 & $15(30)$ \\
\hline 1 & $35(70)$ \\
\hline \multicolumn{2}{|l|}{ Cause of hepatitis } \\
\hline Hepatitis B virus & $42(82)$ \\
\hline Hepatitis $C$ virus & $5(10)$ \\
\hline Alcohol & $2(4.0)$ \\
\hline Other & $1(2.0)$ \\
\hline \multicolumn{2}{|l|}{ Liver cirrhosis } \\
\hline Yes & $41(82)$ \\
\hline No & $9(18)$ \\
\hline \multicolumn{2}{|l|}{ Child-Pugh class } \\
\hline A & $47(94)$ \\
\hline B & $3(6)$ \\
\hline \multicolumn{2}{|l|}{ BCLC stage } \\
\hline $\mathrm{C}$ & $18(36)$ \\
\hline Other & $32(64)$ \\
\hline \multicolumn{2}{|l|}{ Present tumor status } \\
\hline Recurrent & $46(92)$ \\
\hline Primary & $4(8)$ \\
\hline \multicolumn{2}{|l|}{ Tumor size $(\mathrm{cm})$} \\
\hline$\leq 3$ & $46(92)$ \\
\hline$>3$ & $4(8)$ \\
\hline \multicolumn{2}{|l|}{$\alpha$-Fetoprotein (ng/mL) } \\
\hline$<200$ & $47(74)$ \\
\hline$\geq 200$ & $13(26)$ \\
\hline \multicolumn{2}{|c|}{ Portal vein tumor thrombosis } \\
\hline Yes & $10(20)$ \\
\hline No & $40(80)$ \\
\hline \multicolumn{2}{|l|}{ Fraction size } \\
\hline 5 Gy & $45(90)$ \\
\hline 6 Gy & $5(10)$ \\
\hline \multicolumn{2}{|c|}{ Previous treatment (repeated measure) } \\
\hline Surgery & $10(20.8)$ \\
\hline RFA & $13(27.1)$ \\
\hline TACE & $44(91.7)$ \\
\hline RT & $4(8.3)$ \\
\hline Sorafenib & $1(2.1)$ \\
\hline Naïve & $2(4.2)$ \\
\hline
\end{tabular}

ECOG, Eastern Cooperative Oncology Group; BCLC, Barcelona Clinic Liver Cancer; RFA, radiofrequency ablation; TACE, trans-arterial chemoembolization; $\mathrm{RT}$, radiation therapy. 


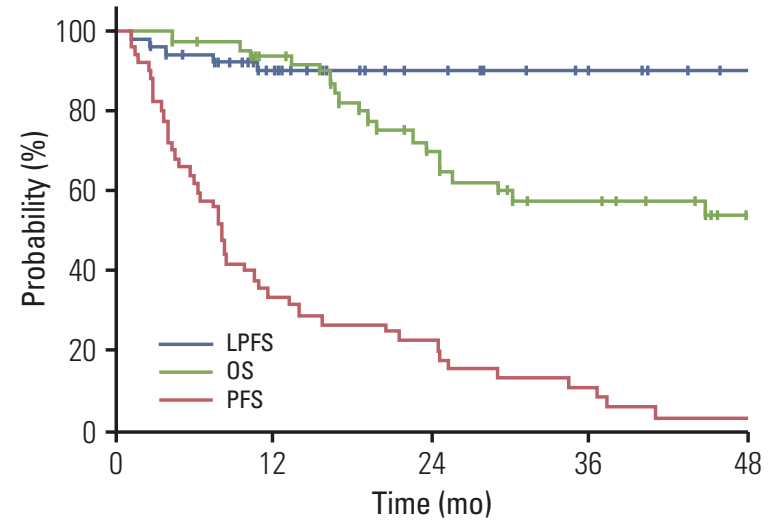

Fig. 1. Kaplan-Meier curves of local progression-free survival (LPFS), progression-free survival (PFS), and overall survival (OS) in all enrolled patients; OS and LPFS were $57.4 \%$ and $89.7 \%$ at 3 years, respectively, but PFS was only $11.2 \%$.

tion size of $6 \mathrm{~Gy}$, complete response was achieved in three patients with one partial response, and one stable disease.

\section{LP and patterns of first site failure}

During follow-up, 46 patients (92\%) progressed to one of several failure patterns. LP was observed in five patients (10\%): two had LP only and three had LP combined with IHP. IHP-only was the most frequent first failure pattern $(n=32,64 \%)$. EHP was observed as a first failure pattern in nine patients (18\%); two patients with EHP also had IHP (Supplementary Fig. S1). The remaining four patients (8\%) showed no evidence of treatment failure throughout the follow-up period.

With the exception of one patient who refused further treatment after EHP, all 45 patients received additional treatment after progression. TACE was repeated for 39 cases, RFA was repeated for nine, and further RT was repeated for 12 patients. Three patients underwent a liver transplant at 4, 7, and 24 months after RT, respectively, and all patients showed a complete pathologic response at the irradiated lesion site.

\section{Survival outcomes}

LPFS, PFS, and OS were $89.7 \%, 11.2 \%$, and $57.4 \%$, respectively, for all enrolled patients 3 years after RT. Kaplan-Meier curves are shown in Fig. 1.

Possible prognostic factors related to survival are shown in Table 2. A maximum tumor size $>3 \mathrm{~cm}$ showed significant association with lower LPFS ( $\mathrm{p}=0.01$ ), but not with decreases in PFS and OS. In contrast, a decrease in AFP 4-8 weeks after RT completion was a significant favorable prognostic factor for PFS only ( $\mathrm{p}=0.02)$. The most important favorable prognostic factor for OS ( $\mathrm{p}=0.03)$, LPFS ( $\mathrm{p}=0.03)$, and PFS ( $\mathrm{p}=0.04$ ) was showing a positive objective response 3 to 5 months after receiving RT. Kaplan-Meier survival curves according to significant prognostic factors are shown in Supplementary Fig. S2. In multivariate analysis with all significant factors and Child-Pugh class, size was the only significant factor on LPFS ( $p=0.009$; hazard ratio [HR], 11.4; 95\% confidence interval [CI], 1.83 to 71.2), AFP decrement on PFS ( $\mathrm{p}=0.02$; HR, $0.50 ; 95 \% \mathrm{CI}, 0.28$ to 0.91 ), and positive objective response on OS ( $\mathrm{p}=0.04$; HR, 0.40 ; $95 \% \mathrm{CI}, 0.17$ to 0.95 ). Positive objective response showed only marginal significance on LPFS ( $\mathrm{p}=0.06)$ and PFS ( $\mathrm{p}=0.07)$.

\section{Treatment-related toxicity}

During treatment, grade I fatigue and/or anorexia were observed in seven patients (14\%), and one patient complained of pre-existing jaundice related to bile-duct tumor invasion that was aggravated during RT.

Treatment-related hematologic and hepatic toxicity profiles of patients within 6 months after receiving RT are shown in Supplementary Table 1. The most common toxicity was elevated liver enzyme levels, which was generally transient and patients typically recovered to normal levels by the next follow-up evaluation.

Four patients $(8 \%)$ with liver cirrhosis and low platelet count before starting RT developed grade III thrombocytopenia $(<50,000$ platelets $/ \mu \mathrm{L})$.

During follow-up, 12 patients (24\%) showed signs of biliary change during their radiologic examinations (Table 3). Among these changes, eight patients were exposed to more than $40 \mathrm{~Gy}$ in the perihilar region, while the other four patients were not. The median time interval between receiving RT and showing initial signs of biliary change was 21.0 months (range, 2.0 to 51.9 months). Three patients developed biloma within 1 month of undergoing TACE treatment. Biliary dilation was observed in nine patients, and the dilation site was the same as the RT field in seven patients. Finally, three patients showed noticeable biliary dilation without definite evidence of tumor progression, and only two patients required intervention for biliary drainage (patients A and B). Although classic RILD was not observed in any patient, four patients $(8 \%)$ showed an elevation of Child-Pugh score of $\geq 2$ points within 6 months.

\section{Significant elevation of total bilirubin after RT}

Four patients $(8 \%)$ showed significant elevation of total bilirubin within 1 year of treatment. Among them, three 
Table 2. Prognostic factors for local progression-free survival

\begin{tabular}{|c|c|c|c|c|c|c|}
\hline Variable & 3-yr LPFS (\%) & p-value & 3-yr PFS (\%) & p-value & 3-yr OS (\%) & p-value \\
\hline \multicolumn{7}{|l|}{ Age $(y r)$} \\
\hline$>60$ & 92.1 & 0.50 & 8.3 & 0.98 & 70.4 & 0.12 \\
\hline$\leq 60$ & 87.0 & & 9.9 & & 40.8 & \\
\hline \multicolumn{7}{|l|}{ Sex } \\
\hline Male & 88.1 & 0.37 & 7.5 & 0.12 & 54.1 & 0.88 \\
\hline Female & 100 & & 38.1 & & 80.0 & \\
\hline \multicolumn{7}{|c|}{ Child-Pugh class } \\
\hline A & 91.1 & 0.11 & 11.2 & 1.00 & 60.2 & 0.14 \\
\hline B & N.R. & & N.R. & & N.R. & \\
\hline \multicolumn{7}{|c|}{ ECOG performance status } \\
\hline 0 & 80.0 & 0.11 & 8.9 & 0.72 & 34.5 & 0.18 \\
\hline 1 & 93.7 & & 9.3 & & 67.0 & \\
\hline \multicolumn{7}{|c|}{$\operatorname{AFP}(\mathrm{ng} / \mathrm{mL})$} \\
\hline$<200$ & 88.8 & 0.77 & 12.6 & 0.56 & 61.6 & 0.40 \\
\hline$\geq 200$ & 92.3 & & 7.7 & & 39.6 & \\
\hline \multicolumn{7}{|l|}{ Size $(\mathrm{cm})$} \\
\hline$\leq 3$ & 93.2 & 0.01 & 12.3 & 0.33 & 59.0 & 0.59 \\
\hline$>3$ & N.R. & & N.R. & & 37.5 & \\
\hline \multicolumn{7}{|c|}{ BCLC stage } \\
\hline Other & 86.7 & 0.42 & 7.8 & 0.62 & 63.3 & 0.30 \\
\hline $\mathrm{C}$ & 94.4 & & 16.7 & & 48.4 & \\
\hline \multicolumn{7}{|l|}{ PVTT } \\
\hline Yes & 100 & 0.24 & 20.0 & 0.21 & 58.3 & 0.86 \\
\hline No & 86.9 & & 9.0 & & 57.0 & \\
\hline \multicolumn{7}{|c|}{ AFP decrement } \\
\hline Yes & 92.5 & 0.40 & 12.0 & 0.02 & 54.1 & 0.28 \\
\hline No & 85.7 & & 9.5 & & 60.1 & \\
\hline \multicolumn{7}{|c|}{ Fraction size } \\
\hline 5 Gy & 88.5 & 0.45 & 9.6 & 0.43 & 54.5 & 0.18 \\
\hline $6 \mathrm{~Gy}$ & 100 & & 30.0 & & 100 & \\
\hline \multicolumn{7}{|c|}{ Objective response } \\
\hline Yes & 94.3 & 0.03 & 12.0 & 0.04 & 66.7 & 0.03 \\
\hline No & 75 & & 8.3 & & 30.0 & \\
\hline
\end{tabular}

LPFS, local progression-free survival; PFS, progression-free survival; OS, overall survival; N.R., not reached; ECOG, Eastern Cooperative Oncology Group; AFP, $\alpha$-fetoprotein; BCLC, Barcelona Clinic Liver Cancer; PVTT, portal vein tumor thrombosis.

patients had a Child-Pugh score elevation of two or more points within six months; one experienced elevation after additional TACE because of LP with IHP; a second had an initial Child-Pugh score of 8 , which worsened due to IHP; and the third received ERBD before RT for treatment of a preexisting stricture of the common bile duct and ERBD was repeated because the first attempt failed 2 months after RT (patient A). The remaining patient with significantly elevated total bilirubin had jaundice during RT, and underwent PTBD insertion 7 months after completing RT (patient B). Patients $A$ and $B$ received 10 fractions of $5 \mathrm{~Gy}$. The patient's PTBD
MRI results before and after RT are shown in Fig. 2. He received hypofractionated $\mathrm{RT}$ directed at the tumor, which had invaded the anterior branch of the right hepatic duct. After 5 months, the patient showed a partial response, however, his bilirubin levels continued to increase, which resulted in a narrowing of the main duct at the conjunction level, as observed on PTBD. 
Table 3. Radiologic biliary change after hypofractionated RT

\begin{tabular}{|c|c|c|c|c|c|c|c|c|}
\hline Patient & Location & $\begin{array}{l}\text { Biliary } \\
\text { change }\end{array}$ & Obstruction & $\begin{array}{c}\text { Interval } \\
(\mathrm{mo})^{\mathrm{a})}\end{array}$ & $\begin{array}{l}\text { Tumor } \\
\text { related }\end{array}$ & $\begin{array}{c}\text { TACE } \\
\text { related }\end{array}$ & $\begin{array}{l}\text { Bilirubin } \\
\text { elevation }\end{array}$ & Intervention \\
\hline A & Perihilar & Dilatation & In-field & 2 & Possible & No & Yes & ERBD re-insertion \\
\hline B & Perihilar & Dilatation & In-field & 7 & Possible & No & Yes & PTBD \\
\hline $\mathrm{C}$ & Non-perihilar & Dilatation & In-field & 32 & No & Possible & No & - \\
\hline $\mathrm{D}$ & Perihilar & Dilatation & In-field & 13 & Definite & No & Yes & PTBD \\
\hline E & Non-perihilar & Dilatation & In-field & 10 & Definite & No & No & - \\
\hline $\mathrm{F}$ & Perihilar & Dilatation & In-field & 21 & Definite & No & No & - \\
\hline G & Perihilar & Dilatation & In-field & 24 & Definite & No & No & - \\
\hline $\mathrm{H}$ & Perihilar & Dilatation & Out-field & 52 & Definite & Possible & No & - \\
\hline I & Non-perihilar & Dilatation & Out-field & 25 & Definite & No & No & - \\
\hline $\mathrm{J}$ & Perihilar & Biloma & Out-field & 20 & No & Definite & No & - \\
\hline K & Perihilar & Biloma & In-field & 6 & No & Definite & No & - \\
\hline $\mathrm{L}$ & Non-perihilar & Biloma & Out-field & 38 & Possible & Definite & No & - \\
\hline
\end{tabular}

RT, radiation therapy; TACE, trans-arterial chemo-embolization; ERBD, endoscopic retrograde biliary drainage; PTBD, percutaneous trans-hepatic biliary drainage. ${ }^{a}$ Time interval of biliary change development after completion of hypofractionated RT.

\section{Discussion}

In terms of patient survival, local tumor control is one of the most important aspects of oncological treatment. In fact, further therapeutic management is almost meaningless in the absence of local control. Likewise, local control is necessary to improve survival in HCC cases, even though IHP will develop during the follow-up period in almost all patients who do not receive a liver transplant [14]. In cases of TACE failure for unresectable HCC patients, there is no other broadly accepted approach for local management; sorafenib is currently the only confirmed treatment option [2,3]. However, response to sorafenib for localized tumor lesions is generally poor [2]. Many investigators are still trying to find a more cost-effective and beneficial modality for local control in unresectable HCC cases.

The recent development of a technique and equipment for performance of RT with respiratory motion management makes it possible to deliver a controllable or even ablative dose to the tumor while introducing an acceptable level of toxicity to the surrounding normal liver tissue [5-7]. Although this was not a randomized study design and the outcome was based on a relatively small number of cases, several prospective and retrospective studies have reported that local control with a higher dose RT, such as SABR, was as high as that received from RFA [6,7]. Based on these results, some radiation-oncology investigators proposed adaption of RT as the next line of management according to the BCLC guidelines [15,16]. In addition, recent guidelines from the Korean Liver Cancer Study Group recommend RT as an alternative modality in cases of unresectable, TACEunresponsive, or refractory HCC [17].

Despite the effectiveness of RT, including SABR, according to multiple prospective as well as retrospective studies, there is still concern regarding RT toxicities in HCC management. Classic RILD, the most serious RT-associated toxicity, has been scarcely reported in recent years due to several predictive models of normal liver toxicity, which are based on previous experience, and advanced RT technology [8]. Gastroduodenal toxicity, another serious complication, has also been extensively studied and can be optimally managed $[18,19]$.

On the other hand, radiation-induced biliary complications, like bile-duct stricture, necrosis, and/or cholangitis, are persistent problems that complicate high-dose RT guidelines $[10,11]$. Radiation-induced biliary complications were reported in early studies on the use of external-beam RT, and were thought to result from injury to the ductal cells and microvessels, leading to progressive microvasculitis and hypoxia [10].

Other modalities besides RT can cause biliary complications in HCC patients. RFA can induce direct thermal injury to the bile duct, and the surgical process, in general, can also cause biliary complications [20,21]. Most frequently, TACE can cause biliary cellular apoptosis and/or necrosis due to hypoxia via occlusion of macro- and/or micro-vessels [22].

In fact, contrary to concerns, biliary complications are currently not common after external hypofractionated RT. In the current study, only two patients (4\%) experienced biliary strictures that could possibly be related to RT. These patients had tumors located in the perihilar region, and the incidence 

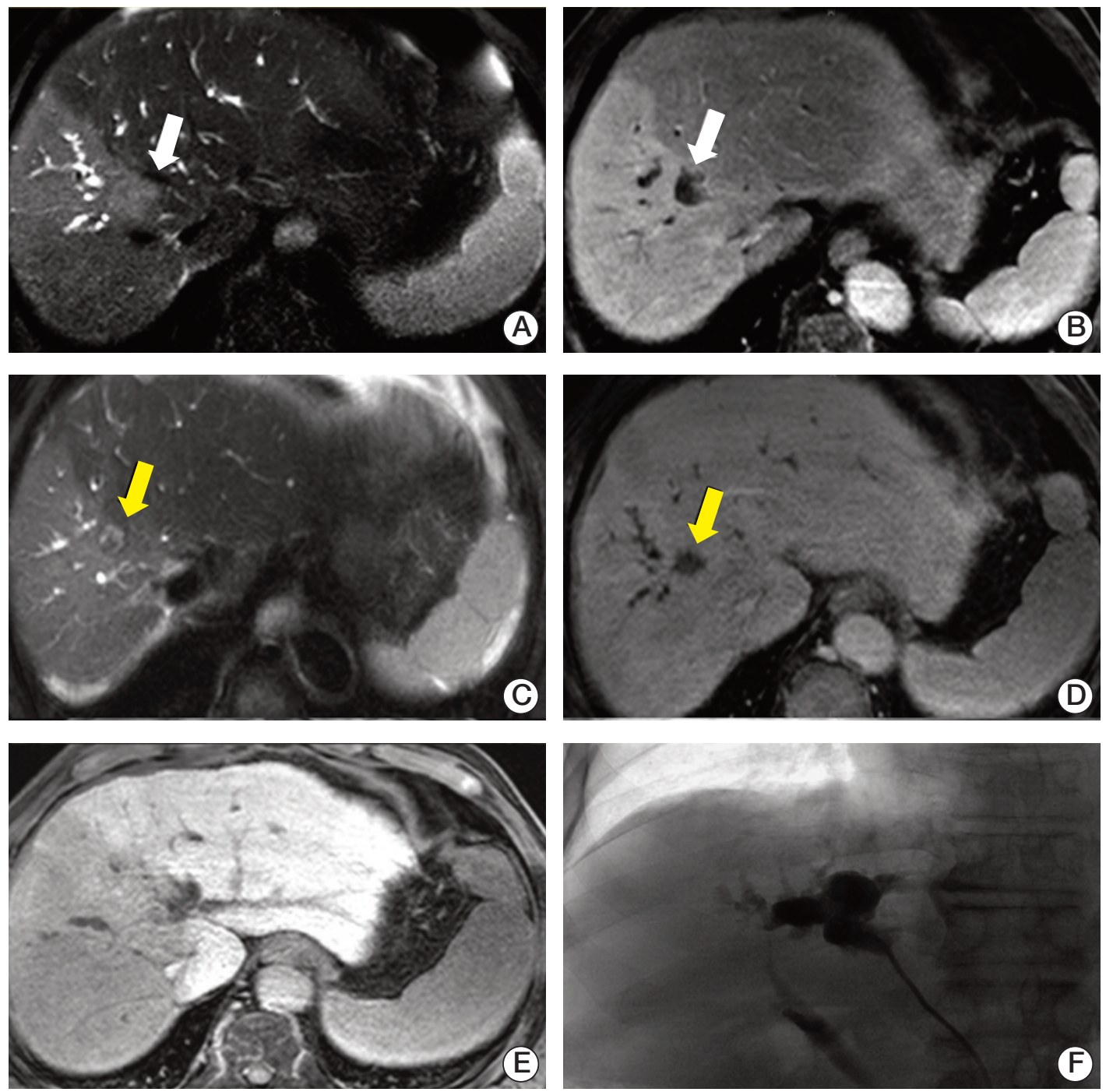

Fig. 2. Biliary stricture after hypofractionated radiation therapy $(\mathrm{RT})$ in unresectable hepatocellular carcinoma. The tumor in segment 8 invaded the bile duct at the hilum side (A, B). The tumor regressed over 4 months following RT (C, D), but the patient experienced concurrent continuous aggravated jaundice $(\mathrm{E})$ and, above the irradiated area, bile duct dilatation was confirmed after percutaneous bile duct drainage (F). Target lesion (white arrow, before RT; yellow arrow, after RT).

of stricture was increased to $7.4 \%$ when focusing on the perihilar region. It is important to note, however, that biliary narrowing and strictures were already present and progressing by the time these patients underwent RT. In addition, there was a possibility that these biliary strictures were related to the primary tumor with or without progression.

The bile-duct complication rate after high-dose RT has been rarely reported in the medical literature [7]. In a previous study, only one of 166 patients experienced biliary stenosis after proton-beam therapy [23] and, in another study, one of 50 patients showed exceptional biliary stenosis after five fractions of 35 to 50 Gy SABR [24]. The complication rate from high-dose hypofractionated RT or SABR was as low as $1 \%-2 \%$, much lower than that of the other standardized modalities described above.

In this study, we analyzed extended follow-up results from patients who received high-dose hypofractionated RT for unresectable, TACE-unresponsive, or refractory HCC. Our results were consistent with previously reported favorable results, indicating that RT offers $>85 \%$ local control and OS was $>55 \%$ at 3 years. In addition, these results are comparable with previous results from TACE and SABR studies 
$[7,25]$.

PFS after high-dose hypofractionated RT was not significantly better than after other treatment modalities: the median PFS was 8 months and approximately $90 \%$ of patients progressed within 3-year post-treatment. As our enrolled patients might have had a higher propensity toward IHP due to a history of multiple management strategies and the presence of recurrent tumors, a direct comparison of PFS outcomes between RT and other modalities was not possible. For these unresectable, TACE-unresponsive, or refractory HCC patients, active control of IHP after high-dose hypofractionated RT might be the most crucial task for improving clinical outcomes. Adjuvant usage of sorafenib could be a good option in this situation; theoretically, these two methods can complement each other for improved HCC management. The Radiation Therapy Oncology Group 1112 will provide valuable information on this topic.

This study had several limitations. Most importantly, there was an unavoidable selection bias due to the retrospective, single institution design, which limits our ability to generalize our findings. In addition, the small number of cases decreased the power of our finding that high-dose hypofractionated RT has a significant effect in treatment of unresectable HCC.

\section{Conclusion}

After long-term follow-up of patients who underwent high-dose hypofractionated RT, the patients' outcomes were comparable to those of patients who received SABR in a different study, indicating that high-dose hypofractionated
RT is a valuable alternative local modality for treatment of unresectable, TACE-unresponsive, or refractory HCC. Biliary complications associated with high-dose hypofractionated RT were minimal and do not present a significant obstacle to treatment plans. A randomized phase III study investigating the efficacy and safety of high-dose RT in unresectable, TACE-unresponsive, or refractory HCC is currently underway.

\section{Electronic Supplementary Material}

Supplementary materials are available at Cancer Research and Treatment website (http://www.e-crt.org).

\section{Conflicts of Interest}

Conflict of interest relevant to this article was not reported.

\section{Acknowledgments}

This research was supported by a Samsung Medical Centergrant (GF01130081) and a grant from Marine Biotechnology Program (20150220) Funded by Ministry of Oceans and Fisheries, Korea.

\section{References}

1. Forner A, Llovet JM, Bruix J. Hepatocellular carcinoma. Lancet. 2012;379:1245-55.

2. Bruix J, Raoul JL, Sherman M, Mazzaferro V, Bolondi L, Craxi A, et al. Efficacy and safety of sorafenib in patients with advanced hepatocellular carcinoma: subanalyses of a phase III trial. J Hepatol. 2012;57:821-9.

3. Ikeda M, Mitsunaga S, Shimizu S, Ohno I, Takahashi H, Okuyama H, et al. Efficacy of sorafenib in patients with hepatocellular carcinoma refractory to transcatheter arterial chemoembolization. J Gastroenterol. 2014;49:932-40.

4. Kim SW, Oh D, Park HC, Lim DH, Shin SW, Cho SK, et al. Transcatheter arterial chemoembolization and radiation therapy for treatment-naive patients with locally advanced hepa- tocellular carcinoma. Radiat Oncol J. 2014;32:14-22.

5. Choi C, Koom WS, Kim TH, Yoon SM, Kim JH, Lee HS, et al. A prospective phase 2 multicenter study for the efficacy of radiation therapy following incomplete transarterial chemoembolization in unresectable hepatocellular carcinoma. Int J Radiat Oncol Biol Phys. 2014;90:1051-60.

6. Yoon SM, Lim YS, Park MJ, Kim SY, Cho B, Shim JH, et al. Stereotactic body radiation therapy as an alternative treatment for small hepatocellular carcinoma. PLoS One. 2013;8:e79854.

7. Jang WI, Kim MS, Bae SH, Cho CK, Yoo HJ, Seo YS, et al. High-dose stereotactic body radiotherapy correlates increased local control and overall survival in patients with inoperable hepatocellular carcinoma. Radiat Oncol. 2013;8:250. 
8. Bujold A, Massey CA, Kim JJ, Brierley J, Cho C, Wong RK, et al. Sequential phase I and II trials of stereotactic body radiotherapy for locally advanced hepatocellular carcinoma. J Clin Oncol. 2013;31:1631-9.

9. Kim TH, Kim DY, Park JW, Kim SH, Choi JI, Kim HB, et al. Dose-volumetric parameters predicting radiation-induced hepatic toxicity in unresectable hepatocellular carcinoma patients treated with three-dimensional conformal radiotherapy. Int J Radiat Oncol Biol Phys. 2007;67:225-31.

10. Cherqui D, Palazzo L, Piedbois P, Charlotte F, Duvoux C, Duron JJ, et al. Common bile duct stricture as a late complication of upper abdominal radiotherapy. J Hepatol. 1994;20: 693-7.

11. Halevy A, Adam A, Stamp G, Benjamin IS, Blumgart LH. Radiation stricture of the biliary ducts: an emerging new entity? HPB Surg. 1992;5:267-70.

12. Bae SH, Park HC, Lim DH, Lee JA, Gwak GY, Choi MS, et al. Salvage treatment with hypofractionated radiotherapy in patients with recurrent small hepatocellular carcinoma. Int J Radiat Oncol Biol Phys. 2012;82:e603-7.

13. Yu JI, Park HC, Lim DH, Kim CJ, Oh D, Yoo BC, et al. Scheduled interval trans-catheter arterial chemoembolization followed by radiation therapy in patients with unresectable hepatocellular carcinoma. J Korean Med Sci. 2012;27:736-43.

14. Sapisochin G, Castells L, Dopazo C, Bilbao I, Minguez B, Lazaro JL, et al. Single HCC in cirrhotic patients: liver resection or liver transplantation? Long-term outcome according to an intention-to-treat basis. Ann Surg Oncol. 2013;20:1194-202.

15. Klein J, Dawson LA. Hepatocellular carcinoma radiation therapy: review of evidence and future opportunities. Int J Radiat Oncol Biol Phys. 2013;87:22-32.

16. Lee IJ, Seong J. Radiotherapeutic strategies in the management of hepatocellular carcinoma. Oncology. 2011;81 Suppl 1: 123-33.

17. Korean Liver Cancer Study Group; National Cancer Center. 2014 Hepatocellular carcinoma practice guideline. Seoul:
Korean Liver Cancer Study Group; 2014.

18. Kim H, Lim DH, Paik SW, Yoo BC, Koh KG, Lee JH, et al. Predictive factors of gastroduodenal toxicity in cirrhotic patients after three-dimensional conformal radiotherapy for hepatocellular carcinoma. Radiother Oncol. 2009;93:302-6.

19. Yoon H, Oh D, Park HC, Kang SW, Han Y, Lim DH, et al. Predictive factors for gastroduodenal toxicity based on endoscopy following radiotherapy in patients with hepatocellular carcinoma. Strahlenther Onkol. 2013;189:541-6.

20. Beltran MM, Marugan RB, Oton E, Blesa C, Nuno J. Accuracy of magnetic resonance cholangiography in the evaluation of late biliary complications after orthotopic liver transplantation. Transplant Proc. 2005;37:3924-5.

21. Giorgio A, Tarantino L, de Stefano G, Coppola C, Ferraioli G. Complications after percutaneous saline-enhanced radiofrequency ablation of liver tumors: 3-year experience with 336 patients at a single center. AJR Am J Roentgenol. 2005;184: 207-11.

22. Miyayama S, Yamashiro M, Okuda M, Yoshie Y, Nakashima $\mathrm{Y}$, Ikeno $\mathrm{H}$, et al. Main bile duct stricture occurring after transcatheter arterial chemoembolization for hepatocellular carcinoma. Cardiovasc Intervent Radiol. 2010;33:1168-79.

23. Chiba T, Tokuuye K, Matsuzaki Y, Sugahara S, Chuganji Y, Kagei K, et al. Proton beam therapy for hepatocellular carcinoma: a retrospective review of 162 patients. Clin Cancer Res. 2005;11:3799-805.

24. Eriguchi T, Takeda A, Sanuki N, Oku Y, Aoki Y, Shigematsu $\mathrm{N}$, et al. Acceptable toxicity after stereotactic body radiation therapy for liver tumors adjacent to the central biliary system. Int J Radiat Oncol Biol Phys. 2013;85:1006-11.

25. Song YG, Shin SW, Cho SK, Choi D, Rhim H, Lee MW, et al. Transarterial chemoembolization as first-line therapy for hepatocellular carcinomas infeasible for ultrasound-guided radiofrequency ablation: a retrospective cohort study of 116 patients. Acta Radiol. 2015;56:70-7. 